University of Wollongong

Research Online

Australian Institute for Innovative Materials -

Papers

Australian Institute for Innovative Materials

$1-1-2019$

\title{
Time-Resolved Imaging of Magnetoelastic Waves by the Cotton-Mouton Effect
}

Tomosato Hioki

Tohoku University

Yusuke Hashimoto

Tohoku University

Tom H. Johansen

University of Wollongong, University of Oslo, tomjo@uow.edu.au

Eiji Saitoh

Tohoku University, Japan Atomic Energy Agency

Follow this and additional works at: https://ro.uow.edu.au/aiimpapers

Part of the Engineering Commons, and the Physical Sciences and Mathematics Commons

Research Online is the open access institutional repository for the University of Wollongong. For further information contact the UOW Library: research-pubs@uow.edu.au 


\title{
Time-Resolved Imaging of Magnetoelastic Waves by the Cotton-Mouton Effect
}

\author{
Abstract \\ We investigate the propagation dynamics of magneto-elastic waves in an out-of-plane (OP) magnetized \\ film using time-resolved magneto-optical (TRMO) microscopy based on the Cotton-Mouton effect. In OP \\ magnetized films, spin precession induces a temporal change in the in-plane component of \\ magnetization $(\mathrm{m})$, which can be measured through birefringence induced by $\mathrm{m}$, or the Cotton-Mouton \\ effect (CME). Here we develop an imaging method based on $\mathrm{CME}$, realizing the observation of stress- \\ driven magneto-elastic waves in an OP magnetized film. This result expands the focus of TRMO \\ microscopy, allowing investigation of magneto-elastic waves in OP magnetized films. \\ Disciplines \\ Engineering | Physical Sciences and Mathematics \\ Publication Details \\ Hioki, T., Hashimoto, Y., Johansen, T. H. \& Saitoh, E. (2019). Time-Resolved Imaging of Magnetoelastic \\ Waves by the Cotton-Mouton Effect. Physical Review Applied, 11 (6), 061007-1-061007-5.
}




\title{
Time-Resolved Imaging of Magnetoelastic Waves by the Cotton-Mouton Effect
}

\author{
Tomosato Hioki, ${ }^{1,}$ * Yusuke Hashimoto, ${ }^{2}$ Tom H. Johansen, ${ }^{3,4}$ and Eiji Saitoh ${ }^{1,2,5,6}$ \\ ${ }^{1}$ Institute for Materials Research, Tohoku University, Sendai 980-8577, Japan \\ ${ }^{2}$ Advanced Institute for Materials Research, Tohoku University, Sendai 980-8577, Japan \\ ${ }^{3}$ Department of Physics, University of Oslo, 0316 Oslo, Norway \\ ${ }^{4}$ Institute for Superconducting and Electronic Materials, University of Wollongong, Northfields Avenue, \\ Wollongong, NSW 2522, Australia \\ ${ }^{5}$ Advanced Science Research Center, Japan Atomic Energy Agency, Tokai 319-1195, Japan \\ ${ }^{6}$ Center for Spintronics Research Network, Tohoku University, Sendai 980-8577, Japan
}

(Received 29 January 2019; revised manuscript received 26 April 2019; published 28 June 2019)

\begin{abstract}
We investigate the propagation dynamics of magneto-elastic waves in an out-of-plane (OP) magnetized film using time-resolved magneto-optical (TRMO) microscopy based on the Cotton-Mouton effect. In OP magnetized films, spin precession induces a temporal change in the in-plane component of magnetization $\left(\mathbf{m}_{\perp}\right)$, which can be measured through birefringence induced by $\mathbf{m}_{\perp}$, or the Cotton-Mouton effect (CME). Here we develop an imaging method based on CME, realizing the observation of stress-driven magnetoelastic waves in an OP magnetized film. This result expands the focus of TRMO microscopy, allowing investigation of magneto-elastic waves in OP magnetized films.
\end{abstract}

DOI: 10.1103/PhysRevApplied.11.061007

Recent development of time-resolved magneto-optical (TRMO) imaging technology has enabled the direct investigation of laser-induced magnetization dynamics in magnetic films with subpicosecond temporal resolution and micrometer spatial resolution [1-4]. This technique allows the direct observation of directional control of spin-wave propagation [5] and the reconstruction of spin-wave dispersions [6,7]. With the TRMO imaging, propagation dynamics and dispersion relation of spin waves has been investigated as one of the key elements in developing spin-wave-based devices [8-14].

In the presence of magnetization and elastic strain coupling, a laser-induced elastic wave can excite magnetization dynamics. Such a coupled wave is called a magnetoelastic wave [7,15-18]. The propagation dynamics of magneto-elastic waves depends on the excitation mechanism driven by laser irradiation [19,20]. Here, the excitation mechanisms include impulsive stimulated Raman scattering (ISRS) [3], inverse Faraday effects (IFEs) [5, 21,22], photoinduced demagnetization, and photoinduced change in the magnetic anisotropy [6,23]. Previous experimental researches on the laser-induced excitation of magneto-elastic waves have been carried out on inplane (IP) magnetized films, where ISRS and photoinduced demagnetization dominantly excite magneto-elastic waves.

\footnotetext{
*hioki.kinken@imr.tohoku.ac.jp
}

In this study, we investigate magnetization dynamics in an out-of-plane (OP) magnetized film by developing a spatio-temporal imaging method using the Cotton-Mouton effect (CME) sensitive to $\mathbf{m}_{\perp}$. In an OP magnetized film, the contribution of photoinduced demagnetization and IFE of which effective fields are parallel to the magnetization of the film is suppressed. Through systematic measurements with changing incident-light polarization and magnetic field, we extract the contributions of the CME from the obtained magneto-optical (MO) images and observe stress-driven magneto-elastic waves in an OP magnetized film. The result expands the focus of TRMO imaging towards OP magnetized films, allowing the investigation of magnetization dynamics excited by laser-induced stress.

We use a Bi-doped lutetium-iron-garnet (LuIG) film grown on a [001] gadolinium-gallium-garnet (GGG) substrate. Temporal images of the Cotton-Mouton effect are obtained by a pump and probe technique and a rotating analyzer method using a CCD camera (Fig. 1) [1]. We use a 100-fs-duration pulsed-light source with a central wavelength of $800 \mathrm{~nm}$ and a $1-\mathrm{kHz}$ repetition frequency. The beam was split into pump and probe beams. The central wavelength of the probe beam is tuned to $630 \mathrm{~nm}$ with an optical parametric amplifier. The pump beam is linearly polarized along the $z$ axis and focused on the sample surface with a radius of $2 \mu \mathrm{m}$. The probe beam is linearly polarized along the $\mathbf{e}_{\phi}$, defined in Fig. 1, with a GlanTaylor prism and is then weakly focused on the sample surface. The polarization direction of $\phi=0$ is parallel to 


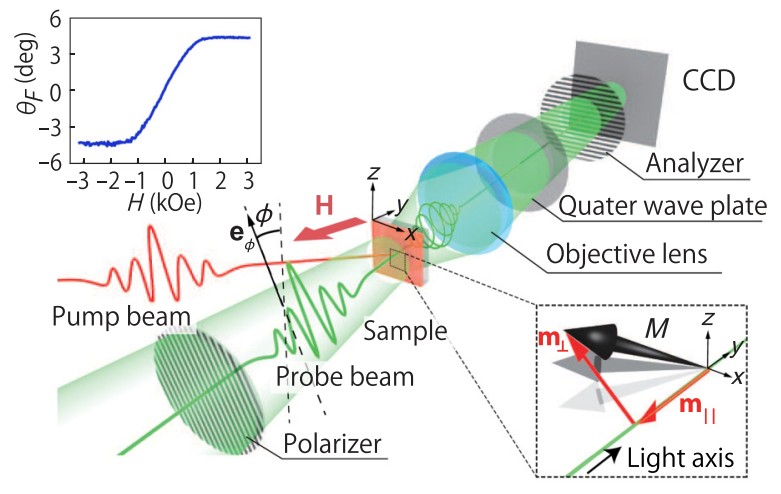

FIG. 1. Schematic illustration of the experimental setup. The pump beam is focused on the sample surface to excite magnetization dynamics. A probe beam is polarized along $\mathbf{e}_{\phi}$ and weakly focused on the sample. The ellipticity of the transmitted probe beam reflects the magnetization component perpendicular to the light axis $\left(\mathbf{m}_{\perp}\right)$. The ellipticity is converted into a rotation angle by a quarter waveplate, and then observed by a rotating analyzer method using a CCD camera [1]. The left top panel shows the magnetic field dependence of the Faraday rotation angle of the sample.

the vertical axis of the field of view. The transmitted probe beam is expanded with an objective lens with a magnification of $\times 20$ and then introduced to a $\mathrm{MO}$ imaging system. The magneto-optical imaging system is composed of a quarter waveplate, an analyzer mounted on a rotation stage, an imaging lens, and a CCD camera. In this setup, obtained images reflect the ellipticity of the transmitted probe beam. The quarter waveplate is used to transform the light ellipticity into the rotation angle of the polarization plane of the light. The magnetization dynamics induced by the pump beam is observed by taking the difference between two images observed with and without the irradiation of the pump beam. The light ellipticity may originate from magnetic circular dichroism (MCD) as well as CME. These two components can be distinguished in terms of their dependence on the probe-beam polarization, since CME depends on the direction of $\mathbf{e}_{\phi}$, while MCD is independent of $\mathbf{e}_{\phi}$.

In this work, we present results from two types of experiments. One is from static measurements of domains magnetized in different directions. The other is on a timeresolved measurements on an OP magnetized film. In the magnetic domain measurement, no external magnetic field is applied and the pump beam did not illuminate the sample. In the time-resolved measurement, we apply an out-of-plane magnetic field of $3.2 \mathrm{kOe}$ to align $\mathbf{M}$ parallel to the sample surface normal. Note that under the magnetic field of $3.2 \mathrm{kOe}$ applied in this experiment, magnetization is saturated. The inset to Fig. 1 shows the magnetic field dependence of $\mathbf{M}$ along the light-beam axis obtained via the Faraday effect. Magnetization dynamics excited by the illuminating pump beam is observed. The temporal change in $\mathbf{m}_{\perp}$ is obtained by scanning the time delay between the pump and probe pulses from $-1.0 \mathrm{~ns}$ to $-13.0 \mathrm{~ns}$ with an increment of $0.1 \mathrm{~ns}$. The origin of the time delay is defined as the time when pump and probe pulses illuminate the sample simultaneously.

Let us first show a static magneto-optical image of magnetic domains observed through CME in Fig. 2(a). The color in the figure indicates the light ellipticity defined in the color code. The sign of the light ellipticity is defined as plus (minus) for a right- (left-)handed elliptically polarized light. The polarization vector of the incident light is $10^{\circ}$ tilted from the $z$ axis $\left(\phi=10^{\circ}\right)$, where the signal intensity is maximum. We see a clear contrast between two domains with different magnetization orientations, as shown in Fig. 2(a). Figure 2(b) shows the ellipticity for each domain as a function of the orientation of the probe-beam polarization $(\phi)$. The obtained signals of the ellipticity for the domains $\mathrm{L}$ and $\mathrm{R}$ are different but the periodicity of the signal is the same with respect to $\phi$. This $\phi$ dependence of the light ellipticity confirms the dominant contribution of the CME.

The $\phi$ dependence of the light ellipticity, shown in Fig. 2(b), is explained by the following model. The CME is birefringence caused by the difference in the refraction index of the light polarized parallel and perpendicular to the magnetization. This induces the phase difference between beams with orthogonal polarizations, and results in the elliptically polarized light [24]. The electric field

(a)

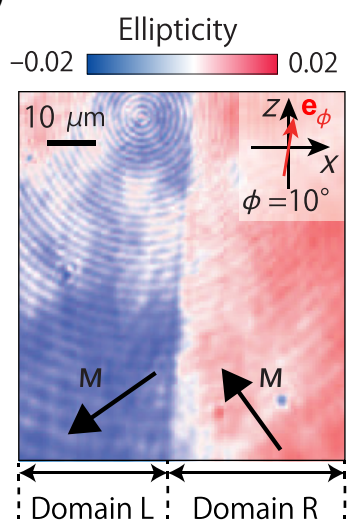

(b)

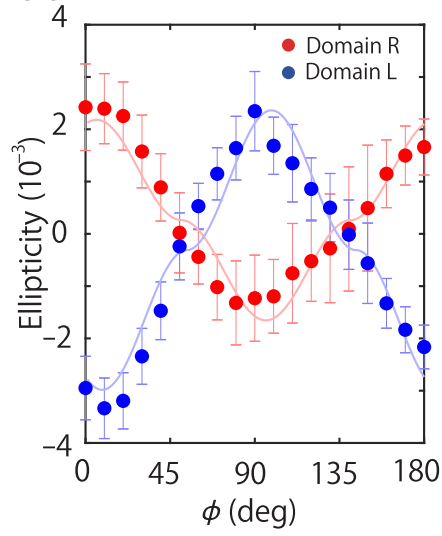

FIG. 2. (a) Static magneto-optical image for magnetic domains with different magnetization orientations. The image is obtained with a probe beam with $\phi=10^{\circ}$. The arrows indicate the orientation of $\mathbf{M}$ in each domain. (b) The probe-beam polarization dependence of the light ellipticity. Red (blue) filled circles represent the data obtained from domain L (R) defined in Fig. 2(a). The solid curves are the fitting results for the $\phi$ dependence of the light ellipticity obtained by using Eq. (1). We take the average of the signals obtained in each domain to improve the signal-to-noise ratio. 
vector of the transmitted probe beam is written as

$$
\mathbf{E}_{\text {out }}=E_{0} e^{i \omega(N \zeta / c)}\left[e^{-i \delta / 2} \cos \phi_{0} \boldsymbol{x}+e^{i \delta / 2} \sin \phi_{0} \boldsymbol{z}\right],
$$

where $\omega, c, N, \zeta, \delta, \phi_{0}$ are the frequency of the transmitted light, the speed of light, the refraction index, the optical path length, the retardation induced by CME, the relative angle between $\mathbf{m}_{\perp}$ and the polarization vector $\mathbf{e}_{\phi}$, respectively. The light ellipticity is defined as the ratio between the intensities of the electric field along two principal axes. Consequently, the ellipticity of the transmitted light is calculated as

$$
\eta_{\mathrm{CME}}=\frac{1-\sqrt{1+\sin ^{2} 2 \phi_{0} \sin ^{2} \delta}}{1+\sqrt{1+\sin ^{2} 2 \phi_{0} \sin ^{2} \delta}} \operatorname{sign}\left[\sin 2 \phi_{0}\right] .
$$

The solid curves in Fig. 2(b) show least-squares fitting for the light ellipticity with Eq. (2). The dominant contribution of the CME in the obtained images is confirmed by the calculated curves, showing good agreement with the experimental data. Two curves overlap when either of them is shifted by $90^{\circ}$, which reveals that the directions of magnetization in the domains $\mathrm{L}$ and $\mathrm{R}$ are normal to each other.

Next, we show the spatio-temporal images of optically excited magnetization dynamics. Figure 3(a) shows the images obtained at the time delay of $5.6 \mathrm{~ns}$ with different probe-beam polarization, indicated by the red arrows in each panel. We define $\vartheta$ as the clockwise angle from the $z$ axis, as shown in Fig. 3(a). We notice that these signals exhibit twofold rotational symmetry and their magnitude is zero along $\mathbf{e}_{\phi}$ or along its normal.

The contribution of CME is confirmed again by the clear $\phi$ dependence of the signal. Figure 3(b) shows the $\phi$ dependence of the light ellipticity at point $P\left(\vartheta=40^{\circ}\right)$. Data are consistent with Eq. (2), which demonstrates that the obtained data corresponds to the in-plane precession component of $\mathbf{M}\left(\mathbf{m}_{\perp}\right)$ in the OP magnetized film. This analysis also clarifies that MCD, independent of $\mathbf{e}_{\phi}$, is negligibly small in these experiments. In addition, we can not see any waves in the Faraday rotation image, reflecting the change in the magnetization in OP orientation as shown in Fig. 3(c). This reveals that the change in $\mathbf{m}_{/ /}$is negligibly small in the experiments.

The signal observed through CME is attributed to the change in $\mathbf{m}_{\perp}$ induced by the magneto-elastic coupling. Magneto-elastic coupling is the coupling between magnetization and elastic strain with a finite wave number [15, $16,25,26]$. The effective field resulting from magneto-elatic coupling is written as follows:

$$
\mathbf{H}_{\mathrm{mec}}^{\mathrm{eff}}=\frac{M_{0} \lambda k}{2 \rho_{0}} \delta_{\theta u_{r}} \mathbf{e}_{k},
$$

where $\lambda, k, M_{0}, \rho_{0}, u_{r}(k), \delta_{\theta}, M_{0}, \mathbf{e}_{k}$ are the gyromagnetic ratio, the volume-magnetostriction constant, wave number, saturation magnetization, mass density, the lattice displacement in the radial orientation, small deviation angle between the surface normal and the light axis, saturation magnetization, and the unit vector parallel to the wavevector, respectively. The consequent change in the radial and azimuth orientation components of the magnetization $\left(\Delta m_{\perp r}\right.$ and $\left.\Delta m_{\perp \phi}\right)$ with infinitesimal time $\Delta t$ can be obtained from an equation of motion of lattice and spin systems with magneto-elastic coupling. $\Delta m_{\perp r}$ and $\Delta m_{\perp \phi}$ are written as $[3,15,25]$

$$
\Delta m_{\perp r}=0, \quad \Delta m_{\perp \phi}=\frac{\gamma M_{0}^{2} \lambda k}{2 \rho_{0}} u_{r}(k) \delta_{\theta} \Delta t,
$$

(a)
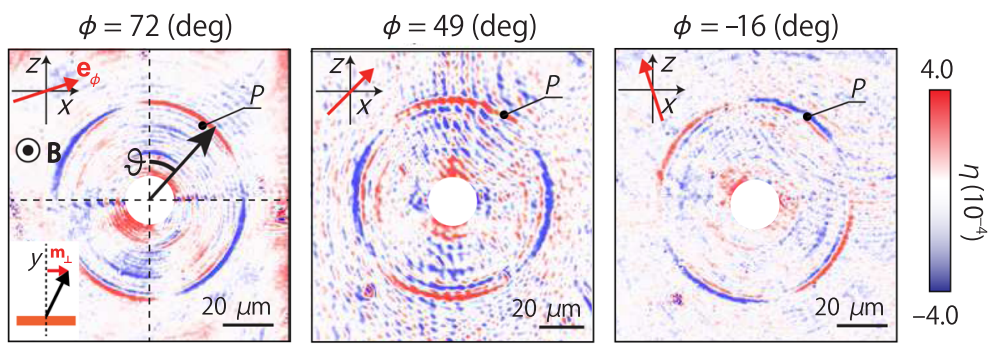

(b)

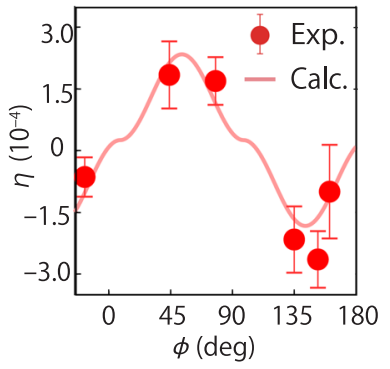

(c)

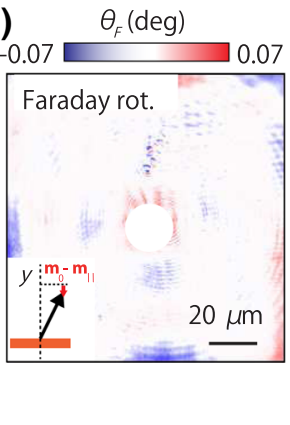

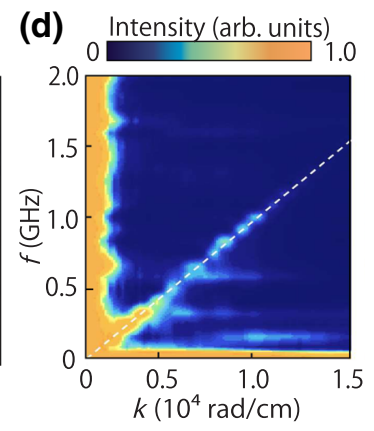

FIG. 3. (a) Time-resolved magnetooptical images obtained by the probe beams with different polarization angles $\phi .\left(\phi=72^{\circ}, 49^{\circ},-16^{\circ}\right.$ in the left, middle, and right panel, respectively.) The color indicates the ellipticity defined in the color code. The center parts of each image are hidden to improve visibility. (b) The $\phi$ dependence of the ellipticity at point $P$ defined in Fig. 3(a). The solid curve is a fitting result. (c) Time-resolved magneto-optical image observed through the Faraday effect. (d) A spin-wave tomography spectrum obtained from the spatio-temporally-resolved images. To improve the signal-to-noise ratio, the spectrum is averaged over $0 \leq \vartheta<360^{\circ}$. The white dotted line represents the linear dispersion of the longitudinal mode of elastic waves. 
where $\gamma$ is the gyromagnetic ratio. Detail derivation of Eq. (4) is provided within the Supplemental Material [27]. Therefore, $\mathbf{m}_{\perp}$ on the wavefront of the magneto-elastic waves is perpendicular to its wavevector. Following to Eq. (4), the signal disappears when $\mathbf{k}$ is perpendicular (parallel) to $\mathbf{e}_{\phi}$ because magnetization orientation is parallel (perpendicular) to polarization, consistent with the obtained results.

In Fig. 3(d), we show a spin-wave tomography [6] spectrum, obtained by analyzing a series of time-resolved magneto-optical images with the model based on Fourier transform. The spin-wave tomography spectrum reflects magnetization dynamics defined as

$$
m_{i}(\mathbf{k}, \omega)=\chi_{i j}^{\mathrm{R}}(\mathbf{k}, \omega) \cdot H_{j}(\mathbf{k}, \omega),
$$

where $\quad m_{i}(\mathbf{k}, \omega)=\mathcal{F}\left[m_{i}(\mathbf{r}, t)\right], \quad \chi_{i j}^{R}(\mathbf{k}, \omega)=\mathcal{F}\left[\chi_{i j}^{R}(\mathbf{r}, t)\right]$, $H_{j}(\mathbf{k}, \omega)=\mathcal{F}\left[H_{j}(\mathbf{r}, t)\right]$ are a magnetization, a retarded susceptibility tensor, and an effective magnetic field acting on local magnetization, respectively, with $\mathcal{F}$ denoting the Fourier transform. $\mathbf{k}$ and $\omega$ are the wavevector and the frequency of the observed waves, respectively. The vector index $i$ represents the in-plane component $(i=x, z)$ because CME is sensitive to $\mathbf{m}_{\perp}$. In Fig. 3(d), we can see a spectral peak along the dashed line, representing the velocity of the wave $\omega / k \simeq 6.5 \mathrm{~km} / \mathrm{s}$. This velocity is consistent with that of the longitudinal mode of magnetoelastic waves in Bi-doped LuIG [6]. Therefore, we attribute the peak showing the linear dispersion in the spectrum to magneto-elastic waves.

In order to confirm that the signal originates not from pure elastic waves but from coupled magneto-elastic waves, in Fig. 4(a), we show spin-wave tomography spectra at different magnetic fields. We find strong magnetic field dependence in the spectrum, showing the magnetic nature in the signal. We model the intensity of the spectrum $I_{m}$ by using a linearlized equation of motion for the coupled lattice and spin system $[15,25,26]$.

$$
\begin{aligned}
I_{m}= & \frac{\gamma M_{0} \sqrt{\omega^{2}+\omega_{s}(H)^{2}}}{\omega_{s}(H)^{2}-\omega^{2}-\omega_{s}(H) \gamma M_{0} \frac{\lambda \lambda_{44}}{2 \rho_{0}\left(\lambda+\lambda_{44}\right)}} \\
& \times \frac{M_{0} \lambda k}{2 \rho_{0}} \delta_{\theta} u_{r}(k),
\end{aligned}
$$

where $\omega, \omega_{s}(H), \lambda, \lambda_{44}$ are the frequency of the longitudinal mode of magneto-elastic waves, the frequency of spin waves, the compressive- and shear-magnetostriction constant, respectively. Detail derivation of Eq. (6) is provided within the Supplemental Material [27]. The intensity of the spectrum decreases when the frequency of elastic waves and spin waves are different at the same wave number. Therefore, spectrum intensity, decreases as magnetic field due to the Zeeman energy, which is observed in Fig. 4(a).
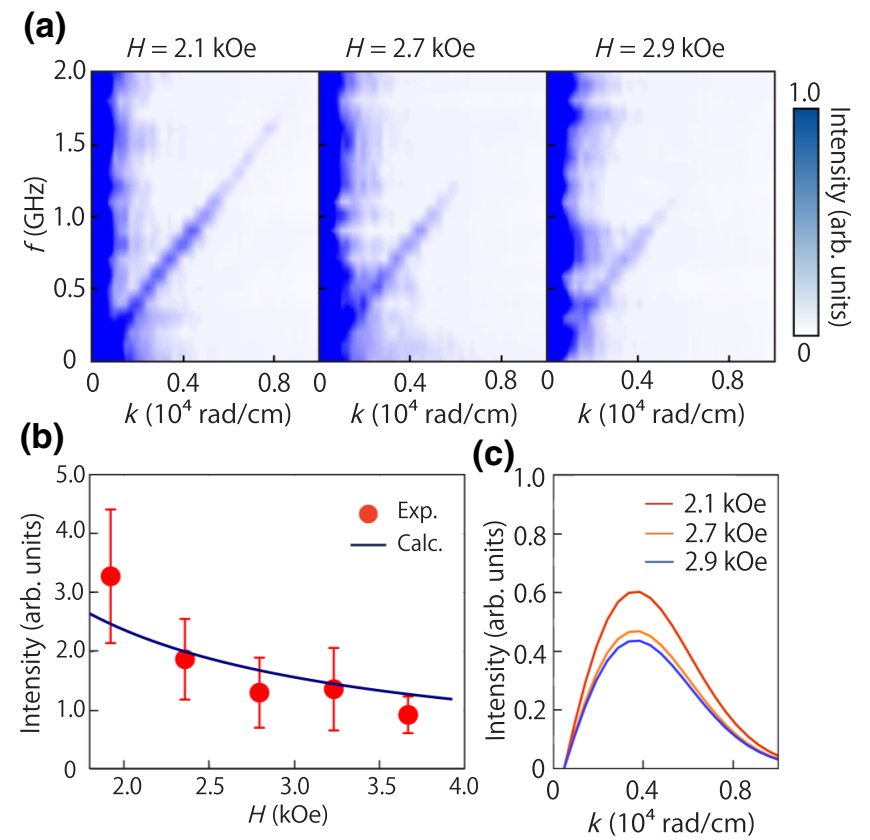

FIG. 4. (a) Spin-wave tomography spectra obtained at different magnetic fields. (b) Intensity of magneto-elastic waves integrated along the line satisfying $\omega=v_{L} k$, where $v_{L}$ is the velocity of longitudinal elastic waves. (c) Precession amplitude factor $I_{m}$ obtained by assuming Gaussian functions for the pump intensity at the focus $\left[u_{r}(k)\right]$ with a radius of $4.8 \times 10^{3}(\mathrm{rad} / \mathrm{cm})$.

Figure 4(b) shows the integrated intensity of the spinwave tomography spectrum shown in Fig. 4(a) satisfying $k<1.0 \times 10^{4}(\mathrm{rad} / \mathrm{cm})$ along the linear dispersion relation. The signal near $k=0$, reflecting the background noise of the images, is excluded. The integrated intensity decreases with external magnetic field. In Fig. 4(c), we plot $I_{m}$ at different magnetic fields assuming that the pump intensity $u_{r}(k)$ is a Gaussian function [7]. The peak intensity of the plot decreases with magnetic field due to the increasing differences in the frequency of spin waves and elastic waves. The solid curve in Fig. 4(b) shows the field dependence of the integrated precession amplitude obtained from Eq. (6). The clear magnetic field dependence of the signal intensity and the agreement with the theory confirms that the signal originates not from pure elastic waves but from the longitudinal mode of magneto-elastic waves.

In summary, we perform time-resolved magneto-optical imaging of the in-plane components of magnetization in a Bi-doped garnet film through the Cotton-Mouton effect. The CME is observed by a rotation analyzer method using a quarter-wave plate, which converts light ellipticity into a rotation angle reflecting the ellipticity of the transmitted light. We carry out static magneto-optical imaging of magnetic domains separated by a $90^{\circ}$ domain wall in an inplane magnetized film and time-resolved magneto-optical imaging for optically excited magnetization dynamics in an out-of-plane magnetized film. We observe propagation 
dynamcis of stress-driven magneto-elastic waves in an out-of-plane magnetized film, taking advantage of CME.

\section{ACKNOWLEDGMENTS}

The authors thank Y. Oikawa and R. Tsuboi for valuable discussions. This work is supported by JST ERATO Grant No. JPMJER1402, Japan, Grant-in-Aid for Scientific Research on Innovative Area "Nano Spin Conversion Science" (JP26103005) from JSPS KAKENHI, Japan. T.H. is supported by JSPS through a research fellowship for young scientists (No. 18J21004) and acknowledges the support from GP-Spin at Tohoku University.

[1] Y. Hashimoto, A. R. Khorsand, M. Savoini, B. Koene, D. Bossini, A. Tsukamoto, A. Itoh, Y. Ohtsuka, K. Aoshima, A. V. Kimel, A. Kirilyuk, and Th. Rasing, Ultrafast timeresolved magneto-optical imaging of all-optical switching in $\mathrm{GdFeCo}$ with femtosecond time-resolution and a $\mu \mathrm{m}$ spatial-resolution, Rev. Sci. Instr. 85, 063702 (2014).

[2] I. Yoshimine, T. Satoh, R. Iida, A. Stupakiewicz, A. Maziewski, and T. Shimura, Phase-controllable spin wave generation in iron garnet by linearly polarized light pulses, J. Appl. Phys. 116, 043907 (2014).

[3] N. Ogawa, W. Koshibae, A. J. Beekmana, N. Nagaosa, M. Kubota, M. Kawasaki, and Y. Tokura, Photodrive of magnetic bubbles via magnetoelastic waves, Proc. Natl. Acad. Sci. USA 88, 10362 (2015).

[4] A. Kamimaki, S. Iihama, Y. Sasaki, Y. Ando, and S. Mizukami, Reciprocal excitation of propagating spin waves by a laser pulse and their reciprocal mapping in magnetic metal films, Phys. Rev. B 96, 014438 (2017).

[5] T. Satoh, Y. Terui, R. Moriya, B. A. Ivanov, K. Ando, E. Saitoh, T. Shimura, and K. Kuroda, Nat. Photon. 6, 662 (2012).

[6] Y. Hashimoto, S. Daimon, R. Iguchi, Y. Oikawa, K. Shen, K. Sato, D. Bossini, Y. Tabuchi, T. Satoh, B. Hillebrands, G. E. W. Bauer, T. H. Johansen, A. Kirilyuk, T. Rasing, and E. Saitoh, All-optical observation and reconstruction of spin wave dispersion, Nat. Comm. 8, 15859 (2017).

[7] Y. Hashimoto, D. Bossini, T. H. Johansen, E. Saitoh, A. Kirilyuk, and T. Rasing, Frequency and wavenumber selective excitation of spin waves through coherent energy transfer from elastic waves, Phys. Rev. B 97, 140404(R) (2018).

[8] A. V. Chumak, V. I. Vasyuchka, A. A. Serga, and B. Hillebrands, Magnon spintronics, Nat. Phys. 11, 453 (2015).

[9] S. Klingler, P. Pirro, T. Brächer, B. Leven, B. Hillebrands, and A. V. Chumak, Design of a spin-wave majority gate employing mode selection, Appl. Phys. Lett. 105, 152410 (2014).

[10] A. V. Chumak, A. A. Serga, and B. Hillebrands, Magnon transistor for all-magnon data processing, Nat. Commun. 5, 4700 (2014).

[11] K. Vogt, F. Y. Fradin, J. E. Pearson, T. Sebastian, S. D. Bader, B. Hillebrands, A. Hoffmann, and H. Schultheiss,
Realization of a spin-wave multiplexer, Nat. Commun. 5, 3727 (2014).

[12] V. E. Demidov, M. P. Kostylev, K. Rott, P. Krzysteczko, G. Reiss, and S. O. Demokritov, Generation of the second harmonic by spin waves propagating in microscopic stripes, Phys. Rev. B 83, 054408 (2011).

[13] V. E. Demidov, S. Urazhdin, and S. O. Demokritov, Control of spin-wave phase and wavelength by electric current on the microscopic scale, Appl. Phys. Lett. 95, 262509 (2009).

[14] A. V. Sadovnikov, C. S. Davies, V. V. Kruglyak, D. V. Romanenko, S. V. Grishin, E. N. Beginin, Y. P. Sharaevskii, and S. A. Nikitov, Spin wave propagation in a uniformly biased curved magnonic waveguide, Phys. Rev. B 96, 060401(R) (2017).

[15] C. Kittel, Interaction of spin waves and ultrasonic waves in ferromagnetic crystals, Phys. Rev. 110, 836 (1958).

[16] L. Dreher, M. Weiler, M. Pernpeintner, H. Huebl, R. Gross, M. S. Brandt, and S. T. B. Goennenwein, Surface acoustic wave driven ferromagnetic resonance in nickel thin films: Theory and experiment, Phys. Rev. B 86, 134415 (2012).

[17] A. E. Clark and R. E. Strakna, Elastic constants of singlecrystal YIG, J. Appl. Phys. 32, 1172 (1961).

[18] A. Rückriegel, P. Kopietz, D. A. Bozhko, A. A. Serga, and B. Hillebrands, Magnetoelastic modes and lifetime of magnons in thin yttrium iron garnet films, Phys. Rev. B 89, 184413 (2014).

[19] K. Shen and G. E. W. Bauer, Laser-Induced Spatiotemporal Dynamics of Magnetic Films, Phys. Rev. Lett. 115, 197201 (2015).

[20] S. P. Zeuschner, T. Parpiiev, T. Pezeril, A. Hillion, K. Dumesnil, A. Anane, J. Pudell, L. Willig, M. Rössle, M. Herzog, A. V. Reppert, and M. Bargheer, Tracking picosecond strain pulses in heterostructures that exhibit giant magnetostriction, Struct. Dyn. 6, 024302 (2019).

[21] J. P. Vanderziel, P. S. Pershan, and L. D. Malmstrom, Optically-Induced Magnetization Resulting from the Inverse Faraday Effect, Phys. Rev. Lett. 15, 190 (1965).

[22] A. V. Kimel, A. Kirilyuk, P. A. Usachev, R. V. Pisarev, A. M. Balbashov, and T. Rasing, Ultrafast non-thermal control of magnetization by instantaneous photomagnetic pulses, Nature 435, 655 (2005).

[23] L. A. Shelukhin, V. V. Pavlov, P. A. Usachev, P. Yu. Shamray, R. V. Pisarev, and A. M. Kalashnikova, Ultrafast laser-induced changes of the magnetic anisotropy in a low-symmetry iron garnet film, Phys. Rev. B 97, 014422 (2018).

[24] A. K. Zvezdin and V. A. Kotov, Modern Magnetooptics and Magnetooptical Materials (IOP Publishing Ltd., Bristol and Philadelphia, 1997).

[25] E. Schlöman, Generation of phonons in high-power ferromagnetic resonance experiments, J. Appl. Phys. 31, 1647 (1960).

[26] A. C. Eringen and G. A. Maugin, Electrodynamics of Continua (Springer-Verlag New York Inc., New York, 1990).

[27] See Supplemental Material at http://link.aps.org/supplemen tal/10.1103/PhysRevApplied.11.061007 for more information regarding the derivation process of (4) and (6). The Supplemental Material contains Refs. [6], [17], and [18]. 